Geometry $\&$ Topology Monographs

Volume 1: The Epstein birthday schrift

Pages 493-509

\title{
Hairdressing in groups: a survey of combings and formal languages
}

\author{
SARAH REES
}

\begin{abstract}
A group is combable if it can be represented by a language of words satisfying a fellow traveller property; an automatic group has a synchronous combing which is a regular language. This article surveys results for combable groups, in particular in the case where the combing is a formal language.
\end{abstract}

AMS Classification 20F10, 20-04, 68Q40; 03D40

Keywords Combings, formal languages, fellow travellers, automatic groups

Dedicated to David Epstein on the occasion of his 60th birthday

\section{Introduction}

The aim of this article is to survey work generalising the notion of an automatic group, in particular to classes of groups associated with various classes of formal languages in the same way that automatic groups are associated with regular languages.

The family of automatic groups, originally defined by Thurston in an attempt to abstract certain finiteness properties of the fundamental groups of hyperbolic manifolds recognised by Cannon in [12], has been of interest for some time. The defining properties of the family give a geometrical viewpoint on the groups and facilitate computation with them; to such a group is associated a set of paths in the Cayley graph of the group (a 'language' for the group) which both satisfies a geometrical 'fellow traveller condition' and, when viewed as a set of words, lies in the formal language class of regular languages. (A formal definition is given in section 2.) Epstein et al.'s book [15] gives a full account; the papers [3] and [16] are also useful references (in particular, [16] is very readable and non-technical).

The axioms of an automatic group are satisfied by all finite groups, all finitely generated free and abelian groups, word hyperbolic groups, the fundamental 
groups of compact Euclidean manifolds, and of compact or geometrically finite hyperbolic manifolds [15, 26], Coxeter groups [10], braid groups, many Artin groups [13, 14, 28, 24], many mapping class groups [27], and groups satisfying various small cancellation conditions [18]. However some very interesting groups are not automatic; the family of automatic groups fails to contain the fundamental groups of compact 3-manifolds based on the Nil or Sol geometries, and, more generally, fails to contain any nilpotent group (probably also any soluble group) which is not virtually abelian. This may be surprising since nilpotent groups have very natural languages, with which computation is very straightforward.

A family of groups which contains the fundamental groups of all compact, geometrisable 3-manifolds was defined by Bridson and Gilman in [9], through a weakening of both the fellow traveller condition and the formal language requirement of regularity for automatic groups. The fellow traveller condition was replaced by an asynchronous condition of the same type, and the regularity condition by a requirement that the language be in the wider class of 'indexed languages'. The class of groups they defined can easily be seen to contain a range of nilpotent and soluble groups.

Bridson and Gilman's work suggests that it is sensible to examine other families of groups, defined in a similar way to automatic groups with respect to other formal language classes. This paper surveys work on this theme. It attempts to be self contained, providing basic definitions and results, but referring the reader elsewhere for fuller details and proofs. Automatic groups are defined, and their basic properties described in section 2; the more general notion of combings is then explained in section 3. A basic introduction to formal languages is given in section 4 for the sake of the curious reader with limited experience in this area. (This section is included to set the results of the paper into context, but all or part of it could easily be omitted on a first reading.) Section 5 describes the closure properties of various classes of combable groups, and section 6 gives examples (and non-examples) of groups with combings in the classes of regular, context-free, indexed and real-time languages.

Acknowledgment The author would like to thank the Fakultät für Mathematik of the Universität Bielefeld for its warm hospitality while this work was carried out, and the Deutscher Akademischer Austauschdienst for financial support. 


\section{Automatic groups}

Let $G$ be a finitely generated group, and $X$ a finite generating set for $G$, and define $X^{-1}$ to be the set of inverses of the elements of $X$. We define a language for $G$ over $X$ to be a set of words over $X$ (that is, products in the free monoid over $X \cup X^{-1}$ ) which maps onto $G$ under the natural homomorphism; such a language is called bijective if the natural map is bijective.

The group $G$ is automatic if it possesses a language satisfying two essentially independent conditions, one a geometric 'fellow traveller condition', relating to the Cayley graph $\Gamma$ for $G$ over $X$, the other a restriction on the computational complexity of the language in terms of the formal language class in which the language lives. Before a precise definition of automaticity can be given, the fellow traveller condition needs to be explained.

Figure 1 gives an informal definition of fellow travelling; we give a more formal definition below. In the figure, the two pairs of paths labelled 1 and 2, and

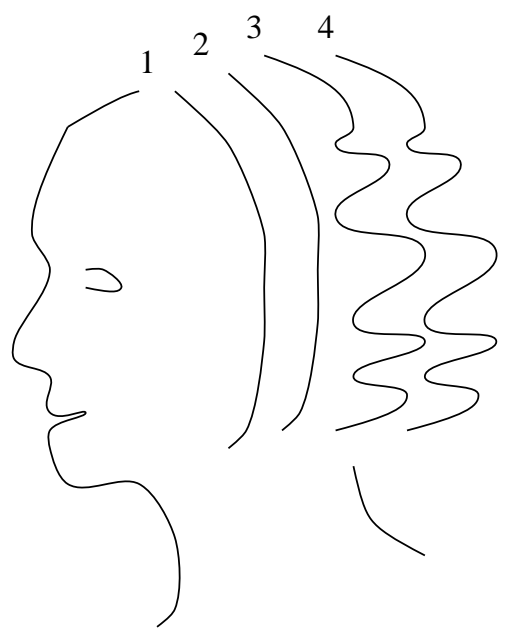

Figure 1: Fellow travellers

3 and 4 synchronously fellow travel at a distance approximately equal to the length of the woman's nose; the pair of paths labelled 2 and 3 asynchronously fellow travel at roughly the same distance. Particles moving at the same speeds along 1 and 2, or along 3 and 4, keep abreast; but a particle on 3 must move much faster than a particle on 2 to keep close to it.

More formally let $\Gamma$ be the Cayley graph for $G$ over $X$. (The vertices of $\Gamma$ correspond to the elements of $G$, and an edge labelled by $x$ leads from $g$ to $g x$, 
for each $g \in G, x \in X)$. A word $w$ over $X$ is naturally associated with the finite path $\gamma_{w}$ labelled by it and starting at the identity in $\Gamma$. The path $\gamma_{w}$ can be parametrised by continuously extending the graph distance function $d_{\Gamma}$ (which gives edges length 1 ); where $|w|=d_{\Gamma}(1, w)$ is the string length of $w$, for $t \leq|w|$, we define $\gamma_{w}(t)$ to be a point distance $t$ along $\gamma_{w}$ from the identity vertex, and, for $t \geq|w|, \gamma_{w}(t)$ to be the endpoint of $\gamma_{w}$. Two paths $\gamma_{1}$ and $\gamma_{2}$ of $\Gamma$ are said to synchronously $K$-fellow travel if, for all $t \geq 0, d_{\Gamma}\left(\gamma_{1}(t), \gamma_{2}(t)\right) \leq K$, and asynchronously $K$-fellow travel if a strictly increasing positive valued function $h=h_{\gamma_{1}, \gamma_{2}}$ can be defined on the positive real numbers, mapping $\left[0, l\left(\gamma_{1}\right)+1\right]$ onto $\left[0, l\left(\gamma_{2}\right)+1\right]$, so that, for all $t \geq 0, d_{\Gamma}\left(\gamma_{1}(t), \gamma_{2}(h(t))\right) \leq K$.

Precisely, $G$ is automatic if, for some generating set $X, G$ has a language $L$ over $X$ satisfying the following two conditions. Firstly, for some $K$, and for any $w, v \in L$ for which $\gamma_{v}$ and $\gamma_{w}$ lead either to the same vertex or to neighbouring vertices of $\Gamma, \gamma_{v}$ and $\gamma_{w}$ synchronously $K$-fellow travel. Secondly $L$ is regular. A language is defined to be regular if it is the set of words accepted by a finite state automaton, that is, the most basic form of theoretical computer; the reader is referred to section 4 for a crash course on automata theory and formal languages. The regularity of $L$ ensures that computation with $L$ is easy; the fellow traveller property ensures that the language behaves well under multiplication by a generator. Although this is not immediately obvious, the definition of automaticity is in fact independent of the generating set for $G$; that is, if $G$ has a regular language over some generating set satisfying the necessary fellow traveller condition, it has such a language over every generating set.

If $G$ is automatic, then $G$ is finitely presented and has quadratic isoperimetric inequality (that is, for some constant $A$, any loop of length $n$ in the Cayley graph $\Gamma$ can be divided into at most $A n^{2}$ loops which are labelled by relators). It follows that $G$ has soluble word problem, and in fact there is a straightforward quadratic time algorithm to solve that.

If $G$ is automatic, then so is any subgroup of finite index in $G$, or quotient of $G$ by a finite normal subgroup, as well as any group in which $G$ is a subgroup of finite index, or of which $G$ is a quotient by a finite normal subgroup. The family of automatic groups is also closed under the taking of direct products, free products (with finite amalgamation), and HNN extensions (over finite subgroups), but not under passage to arbitrary subgroups, or under more general products or extensions. 


\section{Combings}

In an attempt to find a family of groups which has many of the good properties of automatic groups, while also including the examples which are most clearly missing from that family, we define combable groups, using a variant of the first axiom for automatic groups.

Let $G=\langle X\rangle$ be a finitely generated group with associated Cayley graph $\Gamma$. We define an asynchronous combing, or combing for $G$ to be a language $L$ for $G$ with the property that for some $K$, and for any $w, v \in L$ for which $\gamma_{v}$ and $\gamma_{w}$ lead either to the same vertex or to neighbouring vertices of $\Gamma, \gamma_{v}$ and $\gamma_{w}$ asynchronously $K$-fellow travel; if $G$ has a combing, we say that $G$ is combable. Similarly, we define a synchronous combing to be a language for which an analogous synchronous fellow traveller condition holds; hence automatic groups have synchronous combings. Of course, every synchronous combing is also an asynchronous combing.

In the above definitions, we have no requirement of bijectivity, no condition on the length of words in $L$ relative to geodesic words, and no language theoretic restriction. In fact, the term 'combing' has been widely used in the literature, with various different meanings, and some definitions require some of these properties. Many authors require combings to be bijective; in [15] words in the language are required to be quasigeodesic, and in [17] combings are assumed to be synchronous.

The term 'bicombing' is also fairly widely used in the literature, and so, although we shall not be specifically interested in bicombability here, we give a definition for the sake of completeness. Briefly a bicombing is a combing for which words in the language related by left multiplication by a generator also satisfy a fellow traveller property. Specifically, a combing $L$ is a (synchronous, or asynchronous) bicombing if paths of the form $\gamma_{v}$ and $x \gamma_{w}$ (synchronously, or asynchronously) fellow travel, whenever $\gamma_{v}, \gamma_{w} \in L, x \in X$, and $v={ }_{G} x w$, and where $x \gamma_{w}$ is defined to be the concatenation of $x$ and a path from $x$ to $x w$ following edges labelled by the symbols of the word $\gamma_{w}$. A group is biautomatic if it has a synchronous bicombing which is a regular language.

Most known examples of combings for non-automatic groups are not known to be synchronous; certainly this is true of the combings for the non-automatic groups of compact, geometrisable 3-manifolds found by Bridson and Gilman. However, in recent and as yet unpublished work, Bestvina and N. Brady have constructed a synchronous, quasigeodesic (in fact linear) combing for a nonautomatic group. By contrast, Burillo, in [11], has shown that none of the 
Heisenberg groups

$$
\begin{aligned}
H_{2 n+1}= & \left\langle x_{1}, \ldots x_{n}, y_{1}, \ldots y_{n}, z\right|\left[x_{i}, y_{i}\right]=z, \forall i \\
& {\left.\left[x_{i}, x_{j}\right]=\left[y_{i}, y_{j}\right]=\left[x_{i}, y_{j}\right]=1, \forall i, j, i \neq j\right\rangle }
\end{aligned}
$$

or the groups $U_{n}(\mathbf{Z})$ of $n$ by $n$ unipotent upper-triangular integer matrices can admit synchronous combings by quasigeodesics (all of these groups are asynchronously combable). Burillo's result was proved by consideration of higherdimensional isoperimetric inequalities; the case of $H_{3}$ had been previously dealt with in [15].

Let $G$ be a combable group. Then, by [7] theorem 3.1, $G$ is finitely presented, and, by [7] theorems 4.1 and $4.2, G$ has an exponential isoperimetric inequality; hence $G$ has soluble word problem (see [15], theorem 2.2.5). By [17], if $G$ has a synchronous, 'prefix closed' combing (that is, all prefixes of words in the language are in the language), then $G$ must actually have a quadratic isoperimetric inequality. Note that, by [25] (or see [4]), there are finitely presented class 3 soluble groups which have insoluble word problem, and so certainly cannot be combable.

For a combing to be of practical use, it must at least be recognisable. It is therefore natural to consider combings which lie in some formal language class, or rather, which can be defined by some theoretical model of computation. Automatic groups are associated with the most basic such model, that is, with finite state automata and regular languages. In general, where $\mathcal{F}$ is a class of formal languages we shall say that a group is $\mathcal{F}$-combable if it has a combing which is a language in $\mathcal{F}$. Relevant formal languages are discussed in section 4 .

An alternative generalisation of automatic groups is discussed in [5]. This approach recognises that the fellow traveller condition for a group with language $L$ implies the regularity of the language $L^{\prime}$ of pairs of words in $L$ which are equal in the group or related by right multiplication by a generator, and examines what happens when both $L$ and $L^{\prime}$ are allowed to lie in a wider language class (in this particular case languages are considered which are intersections of context-free languages, and hence defined by series of pushdown automata). Some of the consequences of such a generalisation are quite different from those of the case of combings; for example, such groups need not be finitely presented. 


\section{Hierarchy of computational machines and formal languages}

Let $A$ be a finite set of symbols, which we shall call an alphabet. We define a language $L$ over $A$ to be a set of finite strings (words) over $A$, that is a subset of $A^{*}=\cup_{i \in \mathbf{N}} A^{i}$. We define a computational machine $M$ for $L$ to be a device which can be used to recognise the words in $L$, as follows. Words $w$ over $A$ can be input to $M$ one at a time for processing. If $w$ is in $L$, then the processing of $w$ terminates after some finite time, and $M$ identifies $w$ as being in $L$; if $w$ is not in $L$, then either $M$ recognises this after some time, or $M$ continues processing $w$ indefinitely. We define $L$ to be a formal language if it can be recognised by a computational machine; machines of varying complexity define various families of formal languages.

We shall consider various different types of computational machines. Each one can be described in terms of two basic components, namely a finite set $S$ of states, between which $M$ fluctuates, and (for all but the simplest machines) a possibly infinite memory mechanism. Of the states of $S$, one is identified as a start state and some are identified as accept states. Initially (that is, before a word is read) $M$ is always in the start state; the accept states are used by $M$ to help it in its decision process, possibly (depending on the type of the machine) in conjunction with information retrieved from the memory.

We illustrate the above description with a couple of examples of formal languages over the alphabet $A=\{-1,1\}$, and machines which recognise them.

We define $L_{1}$ to be the language over $A$ consisting of all strings containing an even number of 1 's. This language is recognised by a very simple machine $M_{1}$ with two states and no additional memory. $S$ is the set $\{$ even, odd\}; even is the start state and only accept state. $M_{1}$ reads each word $w$ from left to right, and switches state each time a 1 is read. The word $w$ is accepted if $M_{1}$ is in the state even when it finishes reading $w . M_{1}$ is an example of a (deterministic) finite state automaton.

We define $L_{2}$ to be the language over $A$ consisting of all strings containing an equal number of 1 's and -1 's. This language is recognised by a machine $M_{2}$ which reads an input word $w$ from left to right, and keeps a record at each stage of the sum of the digits so far read; $w$ is accepted if when the machine finishes reading $w$ this sum is equal to 0 . For this machine the memory is the crucial component (or rather, the start state is the only state). The language $L_{2}$ cannot be recognised by a machine without memory. $M_{2}$ is an example of a pushdown automaton. 
A range of machines and formal language families, ranging from the simplest finite state automata and associated regular (sometimes known as rational) languages to the Turing machines and recursively enumerable languages, is described in [23]; a treatment directed towards geometrical group theorists is provided by [19]. One-way nested stack automata and real-time Turing machines (associated with indexed languages and real-time languages respectively) are also of interest to us in this article, and are discussed in $[1,2]$ and in $[29,33]$. We refer the reader to those papers for details, but below we try to give an informal overview of relevant machines and formal languages.

Figure 2 shows known inclusions between the formal language classes which we shall describe.

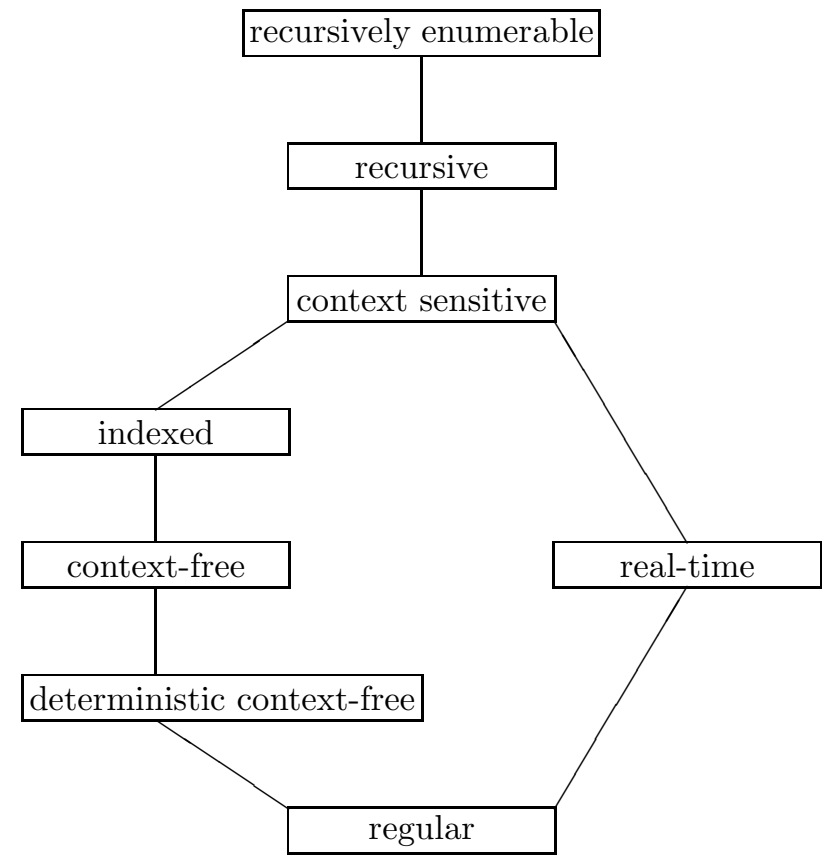

Figure 2: Inclusions between formal language classes

We continue with descriptions of various formal language classes; these might be passed over on a first reading. 


\subsection{Finite state automata and regular languages}

A set of words over a finite alphabet is defined to be a regular language precisely if it is the language defined by a finite state automaton. A finite state automaton is a machine without memory, which moves through the states of $S$ as it reads words over $A$ from left to right. The simplest examples are the so-called deterministic finite state automata. For these a transition function $\tau: S \times A \rightarrow S$ determines passage between states; a word $w=a_{1} \ldots a_{n}\left(a_{i} \in A\right)$ is accepted if for some sequence of states $s_{1}, \ldots s_{n}$, of which $s_{n}$ is an accept state, for each $i$, $\tau\left(s_{i-1}, a_{i}\right)=s_{i}$. Such a machine is probably best understood when viewed as a finite, directed, edge-labelled graph (possibly with loops and multiple edges), of which the states are vertices. The transition $\tau(s, a)=s^{\prime}$ is then represented by an edge labelled by $a$ from the vertex $s$ to the vertex $s^{\prime}$. At most one edge with any particular label leads from any given vertex (but since dead-end non-accept states can easily be ignored, there may be less that $|A|$ edges out of a vertex, and further, several edges with distinct labels might connect the same pair of vertices). A word $w$ is accepted if it labels a path through the graph from the start vertex/state $s_{0}$ to a vertex which is marked as an accept state. Figure 3 gives such a graphical description for the machine $M_{1}$ described at the beginning of section 4 . In such a figure, it is customary to ring the vertices which represent accept states, and to point at the start state with a free arrow, hence the state even is recognisable in this figure as the start state and sole accept state.

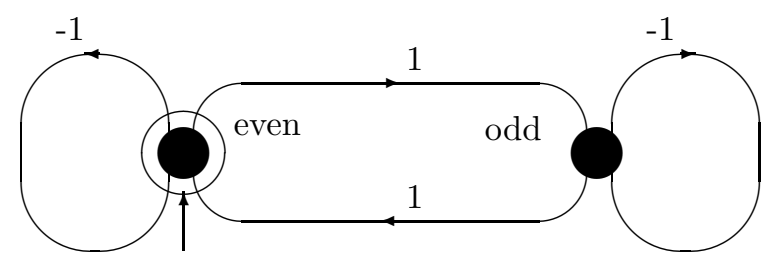

Figure 3: The finite state automaton $M_{1}$

A non-deterministic finite state automaton is defined in the same way as a deterministic finite state automaton except that the transition function $\tau$ is allowed to be multivalued. A word $w$ is accepted if some (but not necessarily all) sequence of transitions following the symbols of $w$ leads to an accept state. The graphical representation of a non-deterministic machine may have any finite number of edges with a given label from each vertex. In addition, further edges labelled by a special symbol $\epsilon$ may allow the machine to leap, without reading from the input string, from one state to another, in a so-called $\epsilon$-move. 
Given any finite state automaton, possibly with multiple edges from a vertex with the same label, possible with $\epsilon$-edges, a finite state automaton defining the same language can be constructed in which neither of these possibilities occur. Hence, at the level of finite state automata, there is no distinction between the deterministic and non-deterministic models. However, for other classes of machines (such as for pushdown automata, described below) non-determinism increases the power of a machine.

\subsection{Turing machines and recursively enumerable languages}

The Turing machines, associated with the recursively enumerable languages, lie at the other end of the computational spectrum from finite state automata, and are accepted as providing a formal definition of computability. In one of the simplest models (there are many equivalent models) of a Turing machine, we consider the input word to be written on a section of a doubly-infinite tape, which is read through a movable tape-head. The tape also serves as a memory device. Initially the tape contains only the input word $w$, the tape-head points at the left hand symbol of that word, and the machine is in the start state $s_{0}$. Subsequently, the tape-head may move both right and left along the tape (which remains stationary). At any stage, the tape-head either reads the symbol from the section of tape at which it currently points or observes that no symbol is written there. Depending on the state it is currently in, and what it observes on the tape, the machine changes state, writes a new symbol (possibly from $A$, but possibly one of finitely many other symbols, or blank) onto the tape, and either halts, or moves its tape-head right or left one position. The input word $w$ is accepted if the machine eventually halts in an accept state; it is possible that the machine may not halt on all input.

Non-deterministic models, where the machine may have a choice of moves in some situations (and accepts a word if some allowable sequence of moves from the obvious initial situation leads it to halt in an accept state), and models with any finite number of extra tapes and tape-heads, are all seen to be equivalent to the above description, in the sense that they also define the recursively enumerable languages.

\subsection{Halting Turing machine and recursive languages}

A halting Turing machine is a Turing machine which halts on all input; thus both the language of the machine and its complement are recursively enumer- 
able. A language accepted by such a machine is defined to be a recursive language.

\subsection{Linear bounded automaton and context sensitive languages}

A linear bounded automaton is a non-deterministic Turing machine whose tapehead is only allowed to move through the piece of tape which initally contains the input word; special symbols, which cannot be overwritten, mark the two ends of the tape. Equivalently (and hence the name), the machine is restricted to a piece of tape whose length is a linear function of the length of the input word. A language accepted by such a machine is defined to be a context sensitive language.

\subsection{Real-time Turing machines and real-time languages}

A real-time Turing machine is most easily described as a deterministic Turing machine with any finite number of doubly-infinite tapes (one of which initially contains the input, and the others of which are initially empty), which halts as it finishes reading its input. Hence such a machine processes its input in 'real time'.

A 'move' for this machine consists of an operation of each of the tape heads, together with a state change, as follows. On the input tape, the tape-head reads the symbol to which it currently points, and then moves one place to the right. On any other tape, the tape-head reads the symbol (if any) to which it currently points, prints a new symbol (or nothing), and then either moves right, or left, or stays still. The machine changes to a new state, which depends on its current state, and the symbols read from the tapes. When the tape-head on the input head has read the last symbol of the input, the whole machine halts, and the input word is accepted if the machine is in an accept state.

A language accepted by such a machine is defined to be a real-time language. $\left\{a^{n} b^{n} c^{n}: n \in \mathbf{N}\right\}$ is an example [33]. Examples are descibed in [33] both of real-times languages which do not lie in the class of context-free languages (described below), and of (even deterministic) context-free languages which are not real-time.

\subsection{Pushdown automata and context-free languages}

A pushdown automaton can be described as a Turing machine with a particu-

larly restricted operation on its tape, but it is probably easier to visualise as 
a machine formed by adding an infinite stack (commonly viewed as a springloaded pile of plates in a canteen) to a (possibly non-deterministic) finite state automaton. Initially the stack contains a single start symbol. Only the top symbol of the stack can be accessed at any time, and information can only be appended to the top of the stack. The input word $w$ is read from left to right. During each move, the top symbol of the stack is removed from the stack, and a symbol from $w$ may be read, or may not. Based on the symbols read, and the current state of the machine, the machine moves into a new state, and a string of symbols (possibly empty) from a finite alphabet is appended to the top of the stack. The word $w$ is accepted if after reading it the machine may be in an accept state. The language accepted by a pushdown automaton is defined to be a context-free language.

The machine $M_{2}$ described towards the beginning of this section can be seen to be a pushdown automaton as follows. The 'sum so far' is held in memory as either a sequence of +1 's or as a sequence of -1 's with the appropropriate sum. When the top symbol on the stack is +1 and a -1 is read from the input tape, the top stack symbol is removed, and nothing is added to the stack. When the top symbol on the stack is -1 and a +1 is read from the input tape, the top stack symbol is removed, and nothing is added to the stack. Otherwise, the top stack symbol is replaced, and then the input symbol is added to the stack. Hence the language $L_{2}$ recognised by $M_{2}$ is seen to be context-free. Similarly so is the language $\left\{a^{n} b^{n}: n \in N\right\}$ over the alphabet $\{a, b\}$. Neither language is regular. For symbols $a, b, c$, the language $\left\{a^{n} b^{n} c^{n}: n \in N\right\}$ is not context-free.

A pushdown automaton is deterministic if each input word $w$ defines a unique sequence of moves through the machine. This does not in fact mean that a symbol of $w$ must be read on each move, but rather that the decision to read a symbol from $w$ at any stage is determined by the symbol read from the stack and the current state of the machine. The class of deterministic context-free languages forms a proper subclass of the class of context-free languages, which contains both the examples of context-free languages given above. The language consisting of all words of the form $w w^{R}$ over some alphabet $A$ (where $w^{R}$ is the reverse of $w$ ) is non-deterministic context-free [23], but is not deterministic context-free.

\subsection{One-way nested stack automata and indexed languages}

A one-way nested stack automaton is probably most easily viewed as a generalisation of a pushdown automaton, that is, as a non-deterministic finite state 
automaton with an attached nest of stacks, rather than a single stack. The input word is read from left to right (as implied by the term 'one-way'). In contrast to a pushdown automaton, the read/write tape-head of this machine is allowed some movement through the system of stacks. At any point of any stack to which the tape-head has access it can read, and a new nested stack can be created; while at the top of any stack it can also write, and delete. The tape-head can move down through any stack, but its upward movement is restricted; basically it is not allowed to move upwards out of a non-empty stack.

The language accepted by a one-way nested stack automaton is defined to be an indexed language. For symbols $a, b, c$, the languages $\left\{a^{n} b^{n} c^{n}: n \in \mathbf{N}\right\}$, $\left\{a^{n^{2}}: n \geq 1\right\},\left\{a^{2^{n}}: n \geq 1\right\}$ and $\left\{a^{n} b^{n^{2}}: n \geq 1\right\}$ are indexed [23], but $\left\{a^{n !}: n \geq 1\right\}$ is not [22], nor is $\left\{\left(a b^{n}\right)^{n}: n \geq 1\right\}[20,22]$.

\section{From one $\mathcal{F}$-combing to another}

Many of the closure properties of the family of automatic groups also hold for other classes of combable groups, often for synchronous as well as asynchronous combings.

In the list below we assume that $\mathcal{F}$ is either the set of all languages over a finite alphabet, or is one of the classes of formal languages described in section 4 , that is that $\mathcal{F}$ is one of the regular languages, context-free languages, indexed languages, context-sensitive languages, real-time languages, recursive languages, or recursively enumerable languages. (These results for all but realtime languages are proved in [9] and [31], and for real-time languages in [21].) Then just as for automatic groups, we have all the following results:

- If $G$ has a synchronous or asynchronous $\mathcal{F}$-combing then it has such a combing over any generating set.

- Where $N$ is a finite, normal subgroup of $G$, and $G$ is finitely generated, then $G$ is synchronously or asynchronously $\mathcal{F}$-combable if and only the same is true of $G / N$.

- Where $J$ is a finite index subgroup of $G$, then $G$ is synchronously or asynchronously $\mathcal{F}$-combable if and only if the same is true of $J$.

- If $G$ and $H$ are both asynchronously $\mathcal{F}$-combable then so are both $G \times H$ and $G * H$. 
A crucial step in the construction of combings for 3-manifold groups in [9] is a construction of Bridson in [8]; combings for $N$ and $H$ can be put together to give an asynchronous combing for a split-extension of the form $N \rtimes H$ provided that $N$ has a combing which is particularly stable under the action of $H$. The set of all geodesics in a word hyperbolic group has that stability, and is a regular language; hence, for any of the language classes $\mathcal{F}$ considered in this section, any split extension of a word hyperbolic group by an $\mathcal{F}$-combable group is $\mathcal{F}$-combable. The free abelian group $\mathbf{Z}^{n}$ also possesses a combing with the necessary stability; hence all split extensions of $\mathbf{Z}^{n}$ by combable groups are asynchronously combable. It remains only to ask in which language class these combings lie.

Stable combings for $\mathbf{Z}^{n}$ are constructed by Bridson in [8] as follows. $\mathbf{Z}^{n}$ is seen embedded as a lattice in $\mathbf{R}^{n}$, and the group element $g$ is then represented by a word which, as a path through the lattice, lies closest to the real line joining the point 0 to the point representing $g$. For some group elements there is a selection of such paths; a systematic choice can clearly be made. It was proved in [9] that $\mathbf{Z}^{2}$ has a combing of this type which is an indexed language; hence all split extensions of the form $\mathbf{Z}^{2} \triangleleft \mathbf{Z}$ were seen to be indexed combable. It followed from this that the fundamental groups of all compact, geometrisable 3-manifolds were indexed combable; for these are all commensurable with free products of groups which are either automatic or finite extensions of $\mathbf{Z}^{2} \rtimes \mathbf{Z}$.

It is unclear whether or not the corresponding combing for $\mathbf{Z}^{n}$ is also an indexed language when $n>2$. Certainly it is a real-time language [21]. Hence many split extensions of the form $\mathbf{Z}^{n} \rtimes H$ are seen to have asynchronous combings which are real-time languages. We give some examples in the final section.

\section{Combing up the language hierarchy}

\subsection{Regular languages}

A group with a synchronous regular combing is, by definition, automatic. More generally, a group with a regular combing is called asynchronously automatic [15]. It is proved in [15] that the asynchronicity of an asynchronously automatic

group is bounded; that is the relative speed at which particles must move along two fellow-travelling words in order to keep apace can be kept within bounds. The Baumslag-Solitar groups

$$
G_{p, q}=\left\langle a, b \mid b a^{p}=a^{q} b\right\rangle
$$


are asynchronously automatic, but not automatic, for $p \neq \pm q$ (see $[15,30]$ ), and automatic for $p= \pm q$.

It is proved in [15] that a nilpotent group which is not abelian-by-finite cannot be asynchronously automatic. From this it follows that the fundamental groups of compact manifolds based on the Nil geometry cannot be asynchronously automatic; N. Brady proved that the same is true of groups of the compact manifolds based on the Sol geometry [6].

\subsection{Context-free languages}

No examples are currently known of non-automatic groups with context-free combings. It is proved in [9] that a nilpotent group which is not abelian-byfinite cannot have a bijective context-free combing; however it remains open whether a context-free combing with more that one representative for some group elements might be possible.

\subsection{Indexed languages}

Bridson and Gilman proved that the fundamental group of every compact geometrisable 3-manifold (or orbifold) is indexed combable. By the results of $[6,15,9]$ described above for regular and context-free combings, this result must be close to being best possible.

It follows immediately from Bridson and Gilman's results that a split extension of $\mathbf{Z}^{2}$ by an indexed combable (and so, certainly by an automatic) group is again indexed combable.

\subsection{Real-time languages}

Since the stable combing of $\mathbf{R}^{n}$ described in section 5 is a real-time language [21], it follows that any split extension over $\mathbf{Z}^{n}$ of a real-time combable group is real-time combable. Hence (see [21]), any finitely generated class 2 nilpotent group with cyclic commutator subgroup is real-time combable, and also any 3 -generated class 2 nilpotent group. Further the free class 2 nilpotent groups, with presentation,

$$
\left\langle x_{1}, \ldots x_{k} \mid\left[\left[x_{i}, x_{j}\right], x_{k}\right], \forall i, j, k\right\rangle,
$$

as well as the $n$-dimensional Heisenberg groups and the groups of $n$-dimensional, unipotent upper-triangular integer matrices, can all be expressed as split 
extensions over free abelian groups, and hence are real-time combable. It follows that any polycyclic-by-finite group (and so, in particular, any finitely generated nilpotent group) embeds as a subgroup in a real-time combable group.

Torsion-free polycyclic metabelian groups with centre disjoint from their commutator subgroup are far from being nilpotent, but are also real-time combable (see [21]). Such groups split over their commutator subgroup, by a theorem of [32]. An example is provided by the group

$$
\left\langle x, y, z \mid y z=z y, y^{x}=y z, z^{x}=y^{2} z\right\rangle
$$

which is certainly not automatic (it has exponential isoperimetric inequality). In fact this group is also indexed combable, since it is of the form $\mathbf{Z}^{2} \triangleleft \mathbf{Z}$.

\section{References}

[1] Alfred V Aho, Indexed grammars - an extension of context-free grammars, J. Assoc. Comp. Mach. 15 (1968) 647-671

[2] Alfred V Aho, Nested stack automata, J. Assoc. Comp. Mach. 16 (1969) 383406

[3] G Baumslag, S M Gersten, M Shapiro, H Short, Automatic groups and amalgams, Journal of Pure and Applied Algebra 76 (1991) 229-316

[4] G Baumslag, D Gildenhuys, R Strebel, Algorithmically insoluble problems about finitely presented solvable groups, Lie and associative algebras. I, Journal of Pure and Applied Algebra 39 (1986) 53-94

[5] Gilbert Baumslag, Michael Shapiro, Hamish Short, Parallel poly pushdown groups, Jorunal of Pure and Applied Algebra, to appear

[6] Noel Brady, The geometry of asynchronous automatic structures on groups, $\mathrm{PhD}$ thesis, UC Berkeley (1993)

[7] Martin R Bridson, On the geometry of normal forms in discrete groups, Proc. London Math. Soc. (3) 67 (1993) 596-616

[8] Martin R Bridson, Combings of semidirect products and 3-manifold groups, Geometric and Functional Analysis 3 (1993) 263-278

[9] MR Bridson, RH Gilman, Formal language theory and the geometry of 3-manifolds, Commentarii Math. Helv. 71 (1996) 525-555

[10] Brigitte Brink, Robert Howlett, A finiteness property of Coxeter groups, Math. Ann. 296 (1993) 179-190

[11] José Burillo, Lower bounds of isoperimetric functions for nilpotent groups, DIMACS Ser. Discrete Math. Theoret. Comput. Sci. (25) 1-8

[12] J W Cannon, The combinatorial structure of cocompact discrete hyperbolic groups, Geom. Dedicata 16 (1984) 123-148

[13] Ruth Charney, Artin groups of finite type are biautomatic, Math. Ann. 292 (1992) 671-683 
[14] Ruth Charney, Geodesic automation and growth functions of Artin groups, Math. Ann. 301 (1995) 307-324

[15] David B A Epstein, J W Cannon, D F Holt, S Levy, M S Patterson, W Thurston, Word processing in groups, Jones and Bartlett, (1992)

[16] Benson Farb, Automatic groups: a guided tour, Enseign. Math. (2) 38 (1992) 291-313

[17] S M Gersten, Bounded cocycles and combings of groups, Internat. J. Algebra Comput. 2 (1992) 307-326

[18] S M Gersten, H B Short, Small cancellation theory and automatic groups, Inv. Math. 102 (1990) 305-334

[19] Robert H Gilman, Formal languages and infinite groups, DIMACS Ser. Discrete Math. Theoret. Comput. Sci. 25 AMS (1996) 27-51

[20] Robert H Gilman, A shrinking lemma for indexed languages, Theoret. Comput. Sci. 163 (1996) 277-281

[21] Robert H Gilman, Derek F Holt, Sarah Rees, Combing nilpotent and polycylic groups, Internat. J. Algebra Comput. to appear

[22] T Hayashi, On derivation trees of indexed grammars, Publ. RIMS Kyoto Univ. 9 (1973) 61-92

[23] John E Hopcroft, Jeffrey D Ullman, Introduction to automata theory, languages and computation, Addison-Wesley, (1979)

[24] Arye Juhász, Large Artin groups are biautomatic, preprint

[25] O G Kharlampovich, A finitely presented group with unsolvable word problem (in Russian), Izv. Akad. Nauk SSSR Ser. Mat. 45 (1981) 852-873

[26] U Lang, Quasigeodesics outside horoballs, Geom. Dedicata 63 (1996) 205-215

[27] Lee Mosher, Mapping class groups are automatic, Annals of Math. 142 (1995) 303-384

[28] David Peifer, Artin groups of extra-large type are automatic, J. Pure Appl. Alg. 110 (1996) 15-56

[29] Michael O Rabin, Real time computation, Israel J. Math. 1 (1963) 203-211

[30] Sarah Rees, Automatic groups associated with word orders other than shortlex, Int. J. Alg. Comp. (to appear)

[31] Sarah Rees, A language theoretic analysis of combings, preprint

[32] Derek J S Robinson, Splitting theorems for infinite groups, Symposia Mathematica 17, Convegni del Novembre e del Dicembre 1973, Academic Press, (1976) 441-470

[33] A Rosenberg, Real-Time Definable Languages, J. Assoc. Comput. Mach. 14 (1967) 645-662

University of Newcastle, Newcastle NE1 7RU, UK

Email: Sarah.Rees@ncl.ac.uk

Received: 15 November 1997 